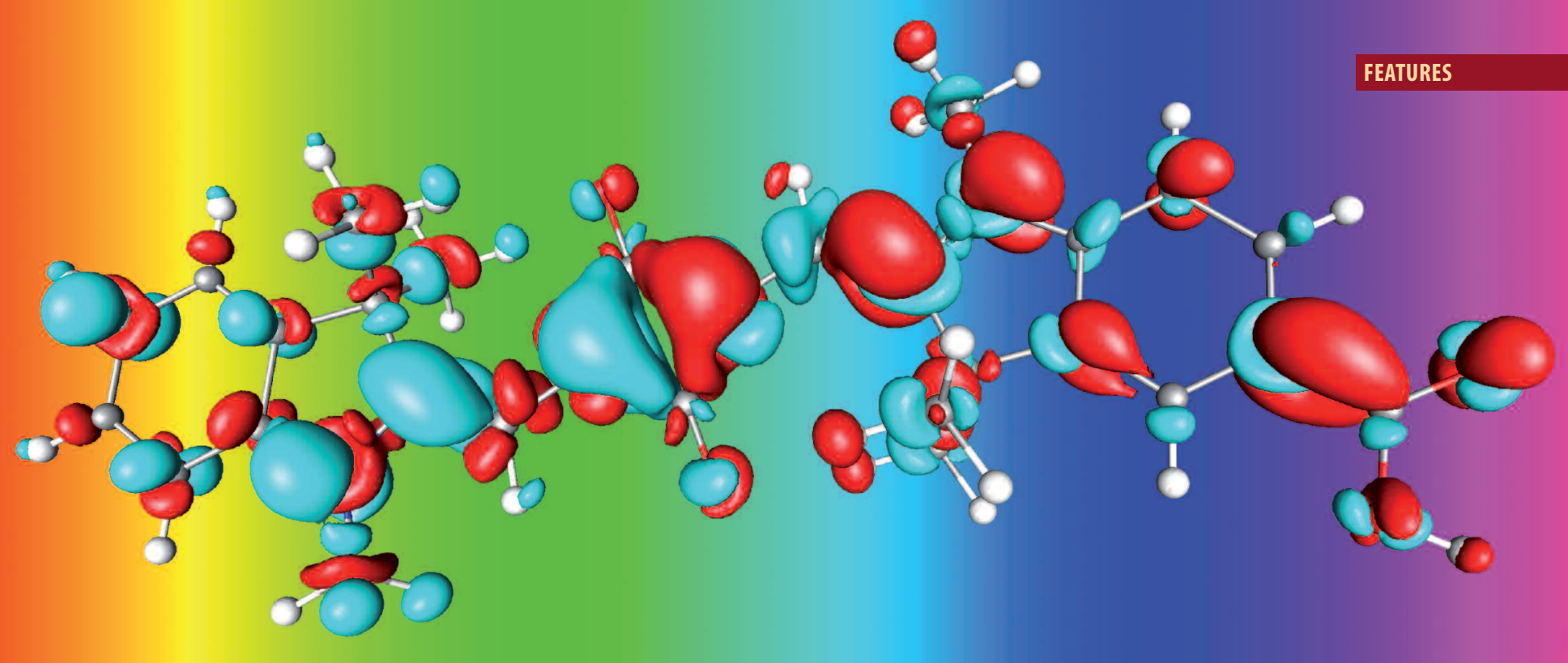

\title{
Sustainable energy: How quantum chemistry can help
}

n Ralph Gebauer - The Abdus Salam International Centre for Theoretical Physics (ICTP), Trieste, Italy - DOI: 10.1051/epn/2011603

\section{Computer simulations of electronic and structural properties can give detailed insight into atomic-scale processes in functional materials. Such studies play an important role in the quest for better strategies to harvest and store renewable energy.}

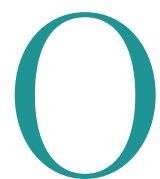
ne of the biggest challenges for science and engineering today is the global energy crisis. According to BP's 2011 Statistical Review of World Energy [1], the worldwide primary energy consumption in 2010 was $503 \times 10^{18} \mathrm{~J}$, which corresponds to an average energy consumption rate of 16 terawatt (TW). Most of this energy is obtained from fossil fuels, with roughly equal parts of oil, coal, and natural gas. Rapid growth of the global population and per capita energy consumption will lead to a considerable projected increase in energy consumption during the next decades. Under moderate assumptions the world energy consumption rate can be forecast to roughly $28 \mathrm{TW}$ in 2050 [2]. Such an increase of the projected energy consumption is in stark contrast with the diminishing availability of affordable fossil fuels. The drastic increase of oil and coal prices in recent years show that the current consumption rate of fossil fuels is pushing the possible extraction rates to its limits. Moreover, the menace of climate change due to the ever-increasing levels of $\mathrm{CO}_{2}$ in the atmosphere calls for a drastic reduction in our use of fossil fuels.

\section{Renewable Energy}

One possible way to address this looming energy crisis is by greatly increasing the use of renewable energy sources like wind, hydro or solar energy. Amongst all those sustainable energy sources, by far the largest resource is the sun. In one hour, about $430 \times 10^{18} \mathrm{~J}$ of solar energy strike the earth, a quantity which is comparable to all the energy which the world is currently consuming in one year. In spite of such a great potential, only a very small fraction of today's energy is provided by the sun, mostly in the form of fuels from biomass. Two main reasons for the low utilization of the sun's potential are the prohibitively large cost of current photovoltaic technologies and the intermittent nature of sunshine.

The issue of the high cost of photovoltaic electricity is generally recognized as a major hurdle for the widespread use of this technology. Traditional photovoltaic panels are usually based on silicon p-n junctions or on thin films of CdTe, a semiconductor. Such panels have seen a strong reduction in price in recent years, mainly due to mass production and the availability of large quantities of pure silicon as raw material. In spite of such progress, the adaptation of this $\triangle$ Excited state of a dye molecule 
v FIG. 1:

Schematic representation of a dye-sensitized solar cell. Solar illumination creates an electronic excitation in dye molecules which are attached to the surface of a wide-bandgap semiconductor.

A typical excitation might be from the dye's highest occupied molecular orbital

(HOMO) to its lowest unoccupied molecular orbital (LUMO).

The excited electron is subsequently injected

into the con-

duction band manifold of the semiconductor. A hole-conducting

electrolyte is used to restore

charge neutrality on the dye and make contact with the second electrode.

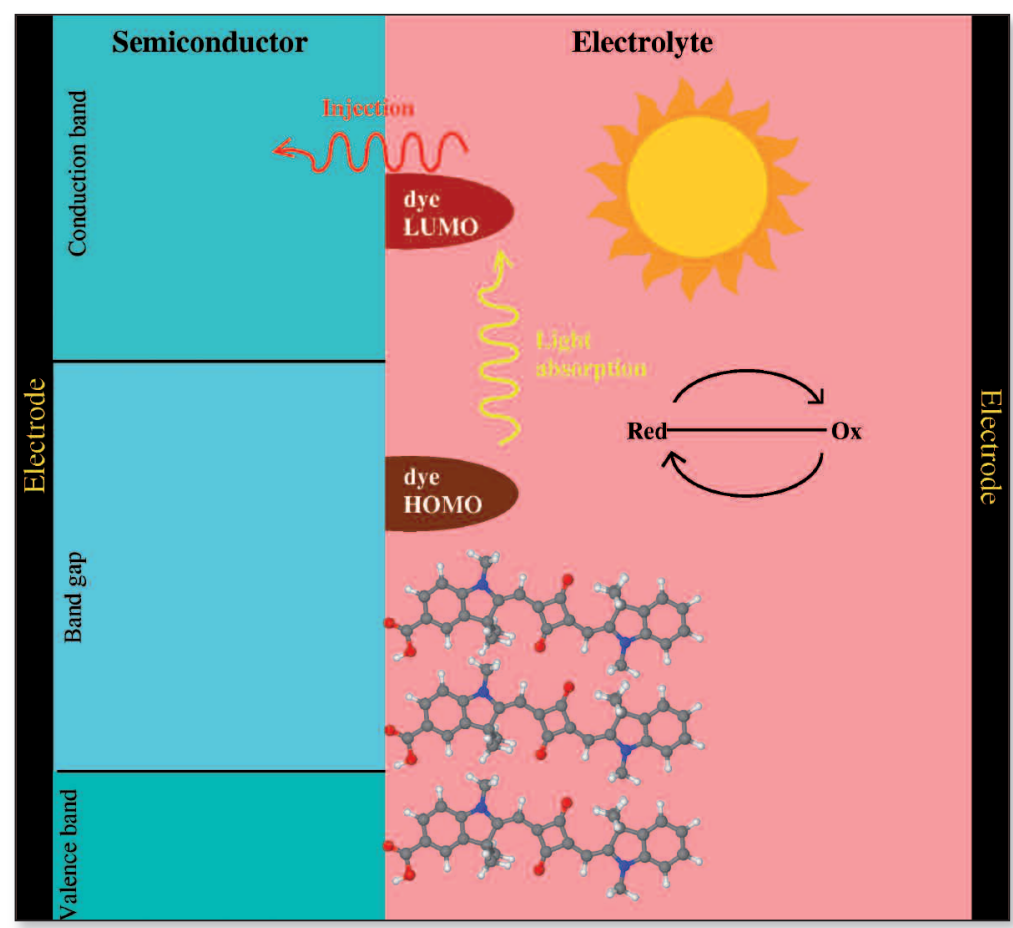

strongly absorb in the visible frequency range. These dye molecules are attached to the surface of a wide-bandgap semiconductor (often $\mathrm{TiO}_{2}$ ). In contrast to traditional solar cells, where the light absorption happens inside the semiconductor, the wide-bandgap material in a DSSC cannot directly harvest solar photons. Instead, the dye molecules act like antennas that capture the sunlight. Following photo-absorption, the excited electron is quickly transferred to the conduction band of the semiconductor from where it is led to an electrode. The hole that the electron leaves behind on the dye molecule is filled from an electrolyte surrounding the semiconductor. The electrolyte is in contact with a second electrode used to close the electrical circuit. This design of a DSSC is depicted schematically in Fig. 1.

Such "third generation" photovoltaic devices do not require ultra-pure, defect-free materials that are expensive to obtain. Dye molecules and the DSSCs can be produced using traditional "wet chemistry" methods. The prospect of low-cost production methods and abundant raw materials are the attractive features of third generation devices. Moreover, efficiencies of up to $11 \%$ have been reported for DSSCs. Nevertheless, much research is still needed to improve this technology and make it a viable alternative to standard solar cells.

Theoretical modeling can intervene at various levels in DSSCs. Firstly, the optical absorption properties of the dye molecules themselves can be computed. Theoretical spectroscopy has always been a core discipline of quantum chemistry. When new dye molecules are considered, preliminary quantum chemical studies can help identify promising candidate molecules. While the synthesis of a new dye can take several months and require well-equipped laboratories, a theoretical spectrum can be obtained within hours, long before the molecule in question has even been produced. In other cases, researchers might consider various ligands to enhance the optical properties of a given molecule. Also in those cases, the availability of computed absorption spectra can guide the choice of ligands.

A second area of theoretical interest is the interaction of the dye molecules with the surface atomic structure. Molecular dynamics simulations can reveal the preferred adsorption sites of molecules and give information about the stability of alternative adsorption geometries. The presence of a liquid electrolyte further complicates the situation. Computer simulations can shed light on the role of the electrolyte in the stability of surface structures and dye adsorption. In Fig. 2 an example is shown where the interaction with liquid water leads to the desorption of a dye from a $\mathrm{TiO}_{2}$ surface [3].

Particular theoretical focus has been drawn to the injection of the excited electron from the dye into the conduction band manifold of the semiconductor. The time scale of this process is a very important factor determining the 
efficiency of the device: if the injection process is slow, the excited electron can de-excite radiatively or thermally towards its ground state and the corresponding energy is lost. Quantum mechanical computations have shown that in some systems the interaction between the excited state on the dye and the semiconductor is strong enough so that the electron injection can occur in a few femtoseconds. This is much faster than typical atomic vibrations, which might lead to alternative de-excitation mechanisms. Moreover, in this case, theoretical insight into the factors determining the injection time can lead to a more targeted choice of surface - dye couples [4].

\section{Energy storage}

One possibility to store the energy of intermittent renewable sources is to convert the energy into chemical bonds. Using $\mathrm{CO}_{2}$ and water as raw materials, an artificial photosynthetic system might produce hydrocarbons that can be easily stored and transported. Various routes to such a system are currently examined. The approaches range from genetically modified forms of algae or bacteria up to electro-catalytic systems where (renewable) electricity is used to produce chemical fuels. One important step in such catalytic processes is the oxidation of water to produce the electrons and protons needed to reduce $\mathrm{CO}_{2}$ to a fuel, as shown schematically in Fig. 3.

The oxidation of water leading to the formation of one oxygen molecule involves four electrons. An efficient catalyst to drive this electrochemical reaction should be such that each one of the four distinct electron transfer steps occurs at nearly the same cost of energy. This is a rather demanding requirement since each step involves chemical species with very distinct oxidation states. Interestingly, nature has managed to satisfy this requirement nearly perfectly in the oxygen-evolving complex of photosystem II [5]. Man-made catalysts, however, suffer from the necessity to apply so-called over-potentials that reduce the efficiency of the water splitting [6].

Quantum chemical simulations can shed light on the involved energy levels and how they depend on the molecular geometry of the catalyst. Theoretical electrocatalytic studies allow exchanging ligands or varying the chemical environment in a controlled manner. Computer simulations can therefore not only lead to a clearer picture of the functioning of a catalyst at the atomic scale, but they can also lead to suggestions on how to improve efficiencies.

\section{Conclusion}

Developing an affordable and reliable energy infrastructure for the future is a formidable task. In spite of the abundant availability of sustainable energy in the form of sunlight, a large-scale switch to solar energy is still hindered by high costs of current technologies and the difficulty to store intermittent electrical energy. Progress

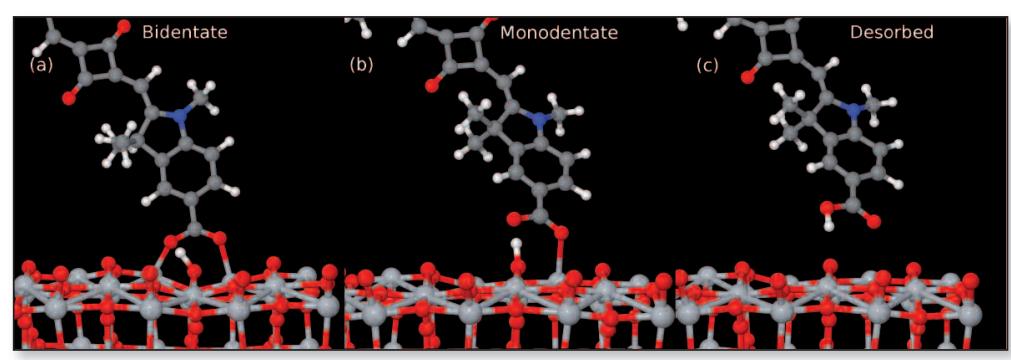

$\triangle$ FIG. 2: Snapshots from a molecular dynamics simulation of the desorption of a dye molecule from a $\mathrm{TiO}_{2}$ semiconductor surface. Panel (a): Initially the dye molecule is attached to the surface. The dye is deprotonated, and the proton (white ball) is attached to a surface oxygen atom (red ball). Panel (b): One of the dye's bonds to the surface is weakened, which leads to the transfer of the surface proton to the molecule and finally to the detachment of the molecule from the surface, see panel (c). The surrounding electrolyte molecules are not shown for clarity.

in these areas can be made possible by bringing together expertise from various fields. In particular, theoretical modelling at the atomic scale is a useful tool for a better understanding of the elementary processes and can lead to a guided development of new materials.

\section{About the author:}

Ralph Gebauer studied physics in Karlsruhe and Lyon. He obtained his PhD from the Ecole Normale Supérieure de Lyon in 1999. After two years as a postdoc at Princeton University, he joined the Abdus Salam International Centre for Theoretical Physics in Trieste in 2002 where he is working as a research scientist in the Statistical Physics and Condensed Matter Section. His research interests lie in atomistic simulations using density-functional theory as well as in computational spectroscopy.

\section{References}

[1] Available online under: www.bp.com/sectionbodycopy.do? categoryld $=7500 \&$ content $\mid d=7068481$

[2] Following scenario B2 in: N. Nakicenovic, R. Swart (Eds.), Special Report on Emissions Scenarios (Intergovernmental Panel on Climate Change, Washington, DC), 48 (2000).

[3] F. De Angelis, S. Fantacci, R. Gebauer, Journal of Physical Chemistry Letters 2, 813 (2011).

[4] W.R. Duncan and O.V. Prezhdo, Annual Review of Physical Chemistry 58, 143 (2007)

[5] J.P. McEvoy and G. W. Brudvig, Chemical Reviews 106, 4455 (2006). [6] H. Dau, C. Limberg, T. Reier, et al., ChemCatChem 2, 724 (2010).
V FIG. 3:

The oxidation of water is an essential step for the storage of energy in form of chemical fuels. This process involves four distinct electron transfer processes. An ideal catalyst should be able to perform each of these steps at the same cost of

energy, as schematically depicted below.

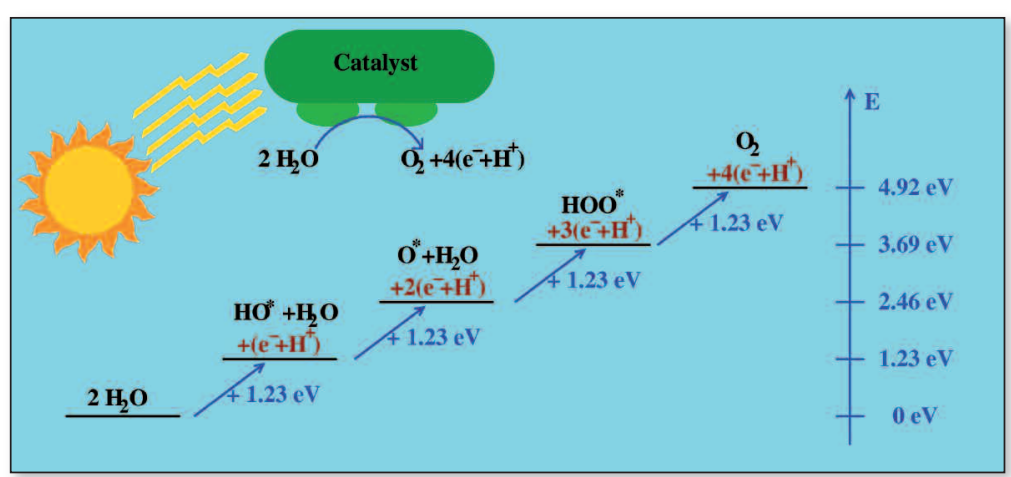

